\title{
Chlorine NQR and Phase Transitions in NOCl*
}

\author{
J. Pirnat ${ }^{\mathrm{a}}$, Z. Trontelj ${ }^{\mathrm{a}}$, and H. Borrmann ${ }^{\mathrm{b}}$ \\ ${ }^{a}$ Institute of Mathematics, Physics and Mechanics, Ljubljana, Slovenia \\ ${ }^{b}$ Max-Planck Institut für Festkörperforschung, Stuttgart, Germany
}

Z. Naturforsch. 51 a, 736-738 (1996); received October 20, 1995

Chlorine NQR studies of solid nitrosyl chloride $\mathrm{NOCl}$ at temperatures below $200 \mathrm{~K}$ were undertaken. They indicate an ionic character of the $\mathrm{N}-\mathrm{Cl}$ chemical bond and confirm the phase transition near $140 \mathrm{~K}$. Thermal hysteresis of the transition temperature was observed.

\section{Introduction}

Nitrosyl chloride $\mathrm{NOCl}$ has previously mainly been studied in the gaseous state by different methods, e. g. the microwave technique $[1,2]$. It is suitable as a model compound for studying the $\mathrm{N}-\mathrm{O}$ and $\mathrm{N}-\mathrm{Cl}$ chemical bonding. The gas phase investigations indicate a considerable degree of ionic character of the $\mathrm{N}-\mathrm{Cl}$ bond [3]. Vibrational spectra in the solid $[4,5]$ show an even increased ionicity, and below $85 \mathrm{~K}$ the appearance of new low frequency Raman lines suggests a solid phase with completely ionized $\mathrm{Cl}$ atoms and NO groups [5].

Because of the reactivity of $\mathrm{NOCl}$, samples are usually kept in sealed glass tubes at lower temperatures. At room temperature the vapor pressure rises to about 2 bars and the compound slowly decomposes. The red liquid crystallizes at $212 \mathrm{~K}$ to ruby-red crystals of space group $\mathrm{Cmcm}$. Upon cooling the color gradually lightens, and at liquid nitrogen temperature the material is said to change into bright-yellow microcrystalline "snow" [5].

The facts mentioned above, and recent X-ray diffraction measurements [6] show that this compound deserves more attention. We have undertaken $\mathrm{Cl}$ NQR investigations in order to learn more about the $\mathrm{N}-\mathrm{Cl}$ and $\mathrm{N}-\mathrm{O}$ chemical bonding in solid $\mathrm{NOCl}$, where there are also indications of interesting structural transformations.

\footnotetext{
* Presented at the XIIIth International Symposium on

Nuclear Quadrupole Interactions,

Providence, Rhode Island, USA, July 23-28, 1995.
}

Reprint requests to Dr. J. Pirnat.

\section{Experimental}

One of us (H. B.) participated in X-ray diffraction measurements of $\mathrm{NOCl}$ powder and single crystals, accompanied by differential thermal analysis and preliminary dielectric measurements [6]. X-ray diffraction measurements and thermal analysis both indicate a phase transition at $142 \mathrm{~K}$. The crystal structure was determined at 8 temperatures between $110 \mathrm{~K}$ and $200 \mathrm{~K}$. At $148 \mathrm{~K}$ and above the space group $\mathrm{Cmcm}$ was observed, whereas at $135 \mathrm{~K}$ and below the crystal symmetry reduces to Pnma. The structure $\mathrm{Cmcm}$ can be represented as a superposition of two mirrorsymmetrical orientations of the NO moiety, occupied with equal probability (see Figure 1). At $138 \mathrm{~K}$ and

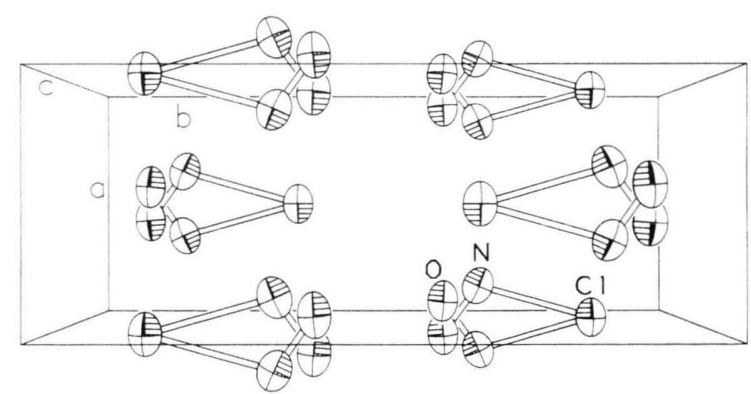

Fig. 1. The disordered crystal structure of $\mathrm{NOCl}$ at $148 \mathrm{~K}$ [6]. Both orientations of the $\mathrm{NO}$ groups are occupied with equal probability.

below the occupation of the two opposite molecular orientations becomes more and more one-sided. The molecular ordering at $138 \mathrm{~K}$ is $85 \%$, and at $113 \mathrm{~K}$ it rises to $97 \%$. Only slight irregularities of the lattice constants can be observed near the transition temperature. We shall call the phase between $142 \mathrm{~K}$ and the 


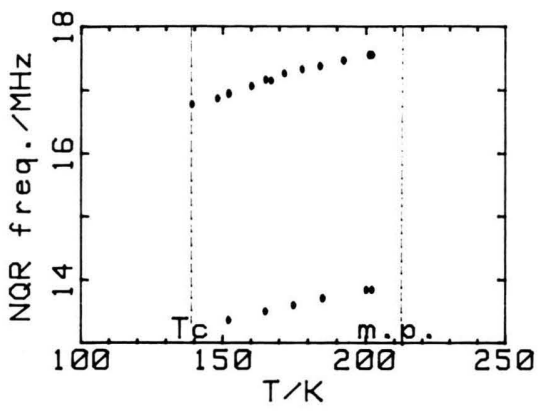

Fig. 2. Temperature dependence of chlorine NQR spectra: upper line: ${ }^{35} \mathrm{Cl}$, lower line: ${ }^{37} \mathrm{Cl}$.

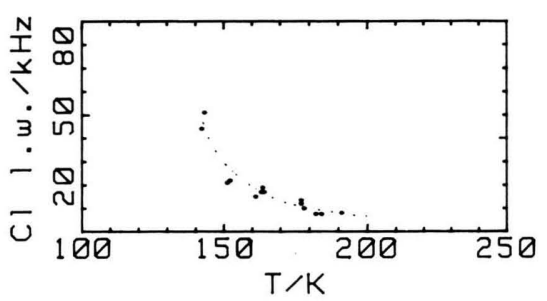

Fig. $3 .{ }^{35} \mathrm{Cl}$ line-width vs. temperature.

melting point the disordered phase, and the one below $142 \mathrm{~K}$ the partly ordered phase.

A strong and narrow ${ }^{35} \mathrm{Cl} \mathrm{NQR}$ line and the corresponding ${ }^{37} \mathrm{Cl} \mathrm{NQR} \mathrm{line} \mathrm{were} \mathrm{found} \mathrm{below} \mathrm{the} \mathrm{melt-}$ ing point in solid $\mathrm{NOCl}$ (at $200 \mathrm{~K} 17.56 \mathrm{Mhz}$ and $13.85 \mathrm{MHz}$ ). To locate the resonance frequencies, a Wilks superregenerative NQR spectrometer was used, while the line-shape and relaxation measurements were done by a Matec pulse NQR spectrometer. The temperature coefficients of both NQR frequencies are positive. On cooling, the line broadens and disappears at $139 \mathrm{~K}$. With increasing temperature from below $139 \mathrm{~K}$, the signal reappears at about $145 \mathrm{~K}$. The temperature dependence of the chlorine NQR spectrum and the ${ }^{35} \mathrm{Cl}$ line-width are shown on Figures 2 and 3. The quadrupole relaxation times $T_{1}$ and $T_{2}$ were measured for ${ }^{35} \mathrm{Cl}$ at different temperatures where the signal was sufficiently strong. The two pulse method gave only the upper limit, which for each relaxation time is as short as $50 \mu \mathrm{s}$. We could not detect any variation of the relaxation times with temperature.

Large frequency intervals were searched for chlorine signals below $142 \mathrm{~K}$, and also below $85 \mathrm{~K}$, where a second phase transition is supposed to be. So far we could not detect any NQR lines.

\section{Discussion}

To describe the possible phase transitions in this compound, the X-ray diffraction measurements [6] suggest the use of the pseudospin formalism: the two possible mirror-symmetrical orientations of the $\mathrm{NOCl}$ molecule are topologically similar to a particle in a double minimum potential and can be represented by the two states of the pseudospin operator. The simplest Hamiltonian describing such a system is [7]

$$
H=-\Omega \sum_{i} S_{i}^{x}-\frac{1}{2} \sum_{i, j} J_{i, j} S_{i}^{z} S_{j}^{z} ;
$$

$S_{i}^{x}, S_{i}^{y}, S_{i}^{z}$ : pseudospin operators $\mathbf{S}=1 / 2, \Omega$ : transverse field - tunneling integral, $J=\sum J_{i, j}$ : longitudinal interaction - intermolecular coupling.

If $2 \Omega<J$, then $\beta_{\mathrm{c}}=\left(k_{\mathrm{B}} T_{\mathrm{c}}\right)^{-1}$ and the critical temperature $T_{\mathrm{c}}$ can be obtained from an equation equivalent to the one from the Ising model [7]:

$$
\frac{2 \Omega}{J}=\tanh \left(\frac{1}{2} \beta_{\mathrm{c}} \Omega\right) .
$$

The phase above $T_{\mathrm{c}}$ is disordered and dynamic, whereas below $T_{\mathrm{c}}$ the structure becomes ordered and frozen.

The NQR signals were so far detected only in the disordered phase ( $142 \mathrm{~K}$ to $212 \mathrm{~K}$ ) which is characterized by the X-ray diffraction with equal occupation of both orientations. Below the temperature where the occupation begins to change (the partly ordered phase), the NQR signal disappeared. The positive slope of the NQR lines temperature dependence reflects the decreasing $\mathrm{N}-\mathrm{Cl}$ bond length observed by the $\mathrm{X}$-ray diffraction. The usual negative slope caused by averaging the EFG by the lattice vibrations (the Bayer mechanism [8]) is probably turned up here by the dominant influence of decreasing ionic and growing covalent character of the bond with increasing temperature.

The disappearance of the signal is considered as an indication of a phase transition for the following reasons: thermal hysteresis of disappearance and reappearance of the chlorine NQR signal when temperature cycling, the characteristic feature of the DTA diagram of the sample and the characteristic behavior of the diffraction diagrams [6]. The existence of the phase transition may be supported also by the emergence of three additional low frequency Raman 
lines between $136 \mathrm{~K}$ and $155 \mathrm{~K}$, although this can be explained in a different way too [5]. The diffraction data indicate the Ising kind of phase transition as the average molecular orientation starts to deviate from zero below the critical temperature. Broad NQR lines are expected as a consequence of only partial molecular orientation and resulting aperiodic crystal structure, until the molecular orientation approaches $100 \%$. This may be the reason for the difficult detection of the chlorine NQR signal. But the signal was not found even far below $T_{\mathrm{c}}$. An Ising type phase transition is also not supported by the constantly short relaxation times in the disordered phase. The latter fact is more compatible with the assumption that both the disordered phase and the partially ordered one have dynamic character with rapid molecular flipping, and the jump appears only in the coherency of the molecular flips when passing the critical temperature from above. This could be the consequence of the coupling of the unit cell dimension (and intermolecular distance) with the intermolecular interaction parameter $J$ in (1).

Far below the Ising phase transition $T_{\mathrm{c}}$, i. e. where the completely ordered structure is frozen, one would expect a sharp NQR spectrum to appear at higher frequencies in the case of unchanged bond

[1] M. A. Roehring and S. G. Kukolich, Mol. Phys. (UK) 76, 221 (1992).

[2] G. Cazzoli, R. Cervellati, and A. M. Mirri, J. Mol. Spectrosc. 56, 422 (1975).

[3] G. Cazzoli, C. Degli Esposti, P. Palmissi, and S. J. Simenone, J. Mol. Spectrosc. 97, 165 (1983).

[4] J. K. McDonald, J. A. Merrit, V. F. Kalasinsky, H. F. Hensel, and J. R. Durig, J. Mol. Spectrosc. 117, 69 (1986). character because the EFG tensor is no more averaged by molecular flipping. On the other hand, if the bond character changes in the sense predicted in [5] (towards an ionic one), the NQR frequencies are drastically lowered and depend mainly on the crystal field. As already mentioned, no NQR lines were found. An other phase transition at $85 \mathrm{~K}$ is reported in [5]. The low temperature structure is difficult to determine because this phase is usually obtained only in microcrystalline form, as mentioned in Section 1. In spite of our efforts we could not find any signal at $77 \mathrm{~K}$ and below, neither at higher frequencies up to $60 \mathrm{MHz}$ nor at low frequencies above $2 \mathrm{MHz}$.

\section{Conclusions}

A chlorine NQR signal, unpublished to our knowledge, has been found in a relatively simple but interesting solid compound convenient to study the chemical bonds as well as the lattice dynamics.

Our measurements have confirmed the phase transition previously found by X-ray measurements and Differential Thermal Analysis.

The absence of the NQR spectrum below $T_{\mathrm{c}}$, especially at much lower temperatures like $77 \mathrm{~K}$ and below, requires further research.

[5] P. M. Killough, B. I. Swanson, and S. F. Agnew, J. Phys. Chem. 93, 7953 (1989).

[6] A. Obermeyer, H. Borrmann, and A. Simon, J. Amer. Chem. Soc. 117, 7887 (1995); H. Borrman, private communication.

[7] R. Blinc, and B. Žekš, Soft Modes in Ferroelectrics and Antiferroelectrics, North-Holland Publ. Comp., Amsterdam, Oxford 1974, Chapter 5.

[8] H. Bayer, Z. Phys. 130, 227 (1951). 